\title{
Localized modes in linear and nonlinear octagonal-diamond lattices with two flat bands
}

\author{
M. G. Stojanović $\odot,{ }^{1}$ M. Stojanović Krasić $\odot,{ }^{2}$ A. Maluckov $\odot,{ }^{1,3}$ M. Johansson $\odot,{ }^{4}$ I. A. Salinas, ${ }^{5}$ \\ R. A. Vicencio $\odot, 5$ and M. Stepić $\oplus^{1}$ \\ ${ }^{1} P^{*}$ Group, Vinča Institute of Nuclear Sciences, University of Belgrade, P.O. Box 522, 11001 Belgrade, Serbia \\ ${ }^{2}$ Faculty of Technology, University of Niš, 16000 Leskovac, Serbia \\ ${ }^{3}$ Center for Theoretical Physics of Complex Systems, Institute for Basic Science (IBS), Daejeon 34126, Korea \\ ${ }^{4}$ Department of Physics, Chemistry and Biology, Linköping University, SE-581 83 Linköping, Sweden \\ ${ }^{5}$ Departamento de Física and MIRO, Facultad de Ciencias Físicas y Matemáticas, Universidad de Chile, Santiago 8370448, Chile
}

(Received 24 April 2020; accepted 29 July 2020; published 28 August 2020; corrected 1 September 2020)

\begin{abstract}
We consider a two-dimensional octagonal-diamond network with a fine-tuned diagonal coupling inside the diamond-shaped unit cell. Its linear spectrum exhibits coexistence of two dispersive bands (DBs) and two flat bands (FBs), touching one of the DBs embedded between them. Analogous to the kagome lattice, one of the FBs will constitute the ground state of the system for a proper sign choice of the Hamiltonian. The system is characterized by two different flat-band fundamental octagonal compactons, originating from the destructive interference of fully geometric nature. In the presence of a nonlinear amplitude (on-site) perturbation, the singleoctagon linear modes continue into one-parameter families of nonlinear compact modes with the same amplitude and phase structure. However, numerical stability analysis indicates that all strictly compact nonlinear modes are unstable, either purely exponentially or with oscillatory instabilities, for weak and intermediate nonlinearities and sufficiently large system sizes. Stabilization may appear in certain ranges for finite systems and, for the compacton originating from the band at the spectral edge, also in a regime of very large focusing nonlinearities. In contrast to the kagome lattice, the latter compacton family will become unstable already for arbitrarily weak defocusing nonlinearity for large enough systems. We show analytically the existence of a critical system size consisting of 12 octagon rings, such that the ground state for weak defocusing nonlinearity is a stable single compacton for smaller systems, and a continuation of a nontrivial, noncompact linear combination of single compacton modes for larger systems. Investigating generally the different nonlinear localized (noncompact) mode families in the semi-infinite gap bounded by this FB, we find that, for increasing (defocusing) nonlinearity the stable ground state will continuously develop into an exponentially localized mode with two main peaks in antiphase. At a critical nonlinearity strength a symmetry-breaking pitchfork bifurcation appears, so that the stable ground state is single peaked for larger defocusing nonlinearities. We also investigate numerically the mobility of localized modes in this regime and find that the considered modes are generally immobile both with respect to axial and diagonal phase-gradient perturbations.
\end{abstract}

DOI: 10.1103/PhysRevA.102.023532

\section{INTRODUCTION}

For several decades, researchers have been interested in the understanding and the experimental observation of transport and localization of energy in various physical media. In particular, solid-state physics uses an atom lattice as a main framework to explore electronic conductivity and insulation [1], which are the key properties for all current technologies. However, the experimental study of those systems has been performed quite indirectly due to the impossibility to image the electronic wave function without affecting its properties. Therefore, during the last decades, several groups have been focused on studying new physical platforms where most of the theoretical predictions could be directly demonstrated and new technologies developed. Specifically flat-band (FB) lattice systems have emerged as the key setups to study transport and localization properties at the linear level [2,3]. A nondiffracting flat band in the linear spectrum is formed by a set of localized spatial states, which occupy a small number of unit cells $[4,5]$. For about five years, this kind of system has been extensively studied on several physical configurations [6-17], with a clear evidence of predicted properties. This naturally has stimulated interest from the theoretical and experimental community and new studies have been conducted considering, for example, distortion of the lattice by including defects or disorder $[18,19]$ and, very recently, extra linear-nonlinear interactions [20] and parity-time symmetries [21].

Although it is possible to experimentally observe diffusion and localization of wave packets on a FB linear lattice [22], the coherent transport of a given localized FB linear state is simply not possible. Therefore, it becomes important to explore different FB models for the possibility of finding coherent mobility through a given lattice, in order to disseminate the energy in a controlled way [23]. Nonlinear effects are a natural way to explore this due to the possibility of localizing energy by using a balance between diffraction and 
self-focusing properties, which generates the so-called discrete solitons [24-26]. Additionally, cubiclike nonlinearities appear naturally when increasing the weight of the wave function and are the common elements on current setups $[8,9,15,16,20]$. Depending on the particular geometry of the lattice, different stability considerations could emerge with respect to fundamental single-site excitations and compactonlike ring modes. In a FB system, due to a perfect cancellation of diffraction though the lattice on a linear level, the addition of nonlinearity may under certain conditions (e.g., amplitude homogeneity [5,27,28] or tuning of coupling coefficients [29]) preserve the compactness of the mode and give a continuous one-parameter family of exact compact nonlinear stationary solutions. The absence of exponentially decaying tails (which typically appear for discrete solitons) increases the difficulty to coherently move a compact FB mode, as there is no tail that could resonate with traveling waves. But, as it has been shown only numerically up to now, it is possible to achieve some control on localized nonlinear solutions on very specific FB systems [27,30-32].

Here we investigate the light propagation through a nonlinear two-dimensional (2D) octagonal-diamond lattice (ODL) with a fine-tuned diagonal coupling constant inside the diamond-shaped unit cells [33], which could be experimentally fabricated by laser-inscribed waveguide arrays in transparent glass [34]. With the diagonal coupling included, the ODL has an eigenvalue spectrum containing two nondispersive bands, touching a dispersive band (DB) at opposite band edges [33]. Therefore, we expect the nonlinear on-site perturbation of the network to provide as well continuation of the linear FB compact states into families of nonlinear modes, as creation of new families of nonlinear localized modes in the gaps inside the eigenspectrum and in the semiinfinite gaps. The octagonal-diamond (also termed "squareoctagon") geometry, without the diagonal coupling leading to flat bands, was considered also in condensed-matter systems in the framework of the topological phase transitions in the Hubbard model [35,36], and those induced by spin-orbit interaction and gauge fields [37]. There, it was related to the appearance of nontrivial nearly FB states with particular topological properties.

The outline of the paper is as follows. In Sec. II the main tools used to model and analyze the ODL system are presented. The linear spectrum is described, with its compact modes [33] as eigenvectors of the FB component manifolds. The technique used for the stability analysis of nonlinear modes, as well as quantities used to characterize their localization and symmetry-breaking properties, are also presented in Sec. II. In Sec. III the possibility for the existence of dynamically stable nonlinear compact localized modes is studied, and the effect of various types of instabilities is illustrated numerically. Special effort is done to investigate the properties of localized modes with frequencies in the semi-infinite gap which in the linear ODL appears above the FB-DB-FB triplet, and in particular the size-dependent nature of the ground state in a regime of weak defocusing nonlinearity is analyzed. Stability and mobility properties of general families of noncompact, localized modes for increasing nonlinearity are also numerically analyzed in Sec. III. Finally, Sec. IV concludes the paper.

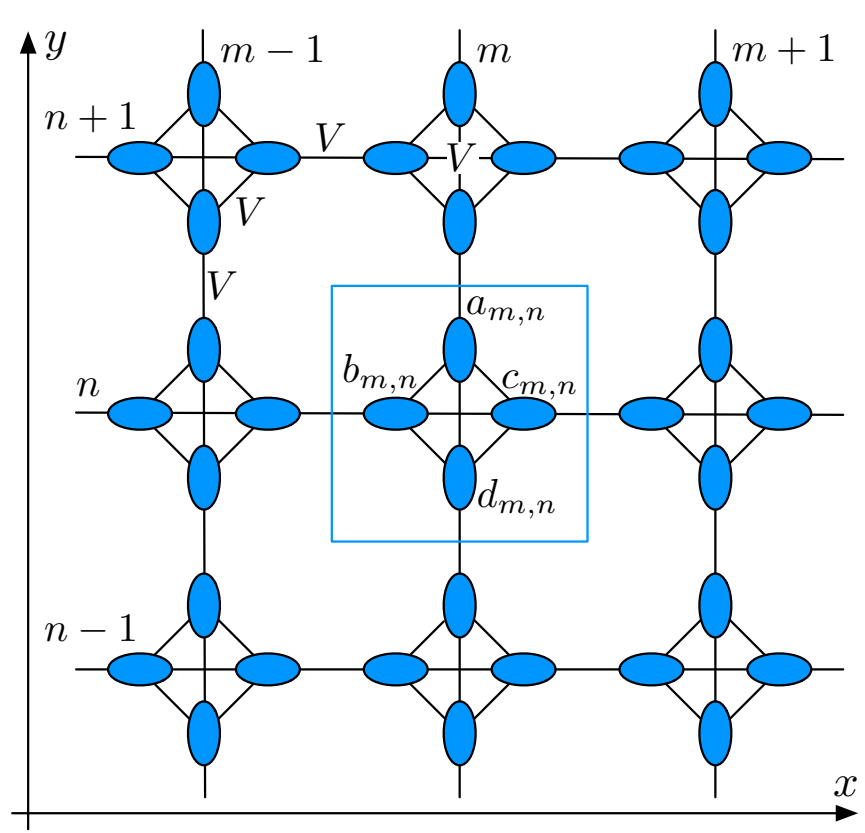

FIG. 1. Schematic representation of an ODL. The unit cell $m, n$ is denoted by a square. The figure includes our physical proposal for making possible that all couplings are considered as equal.

\section{MODEL, LINEAR PROPERTIES, AND NUMERICAL TOOLS}

\section{A. Model equations}

A sketch of the uniform ODL that we consider in this work is presented in Fig. 1. The primitive (unit) cell consists of four sites $(a, b, c, d)$, linearly coupled with each other with the same coupling constant, including two diagonal couplings.

Denoting the total number of unit cells in the $x$ and $y$ directions with $M$ and $N$, respectively, and considering direct linear coupling (represented by lines in figures) and cubic on-site nonlinear coefficients, the 2D ODL can be modeled by a set of $4 M N$ coupled differential equations, constituting a discrete nonlinear Schrödinger-like system with four degrees of freedom per unit cell:

$$
\begin{aligned}
& -i \dot{a}_{m, n}=V\left(b_{m, n}+c_{m, n}+d_{m, n}+d_{m, n+1}\right)+\gamma\left|a_{m, n}\right|^{2} a_{m, n}, \\
& -i \dot{b}_{m, n}=V\left(a_{m, n}+d_{m, n}+c_{m, n}+c_{m-1, n}\right)+\gamma\left|b_{m, n}\right|^{2} b_{m, n}, \\
& -i \dot{c}_{m, n}=V\left(a_{m, n}+d_{m, n}+b_{m, n}+b_{m+1, n}\right)+\gamma\left|c_{m, n}\right|^{2} c_{m, n}, \\
& -i \dot{d}_{m, n}=V\left(b_{m, n}+c_{m, n}+a_{m, n}+a_{m, n-1}\right)+\gamma\left|d_{m, n}\right|^{2} d_{m, n} .
\end{aligned}
$$

Here, $\dot{\xi} \equiv \partial \xi / \partial z(\xi=a, b, c, d)$ with $z$ the normalized propagation coordinate (which on occasions also is referred to as a "time" coordinate below). $a_{m, n}, b_{m, n}, c_{m, n}$, and $d_{m, n}$ are the mode amplitudes at sites $a, b, c$, and $d$ at the $m, n$ lattice position, with $m \in[1, M]$ and $n \in[1, N]$ numbering the unit cells. $V$ is the coupling constant between sites, which we have assumed as symmetric for directly connected neighbors. This is possible by having in mind that experimental waveguides are mostly elliptically oriented [34], so we can consider a rotated distribution of them as sketched in Fig. 1. In this way, long-distance (diagonal) coupling interactions can be 
balanced to have a magnitude equal to that of shorter-distance ones. The effective nonlinear coefficient $\gamma$ represents focusing $(\gamma / V>0)$ or defocusing $(\gamma / V<0)$ Kerr nonlinearities.

Model (1) is characterized by two conserved quantities: the total power (norm) $P$ and the Hamiltonian $H$, defined as

$$
\begin{aligned}
& P=\sum_{m, n}\left(\left|a_{m, n}\right|^{2}+\left|b_{m, n}\right|^{2}+\left|c_{m, n}\right|^{2}+\left|d_{m, n}\right|^{2}\right), \\
H= & \frac{1}{2}\left\{\sum_{m, n} \frac{1}{2} \gamma\left(\left|a_{m, n}\right|^{4}+\left|b_{m, n}\right|^{4}+\left|c_{m, n}\right|^{4}+\left|d_{m, n}\right|^{4}\right)\right. \\
& +V a_{m, n}\left(b_{m, n}+c_{m, n}+d_{m, n}+d_{m, n+1}\right)^{*} \\
& +V b_{m, n}\left(a_{m, n}+d_{m, n}+c_{m, n}+c_{m-1, n}\right)^{*} \\
& +V c_{m, n}\left(a_{m, n}+d_{m, n}+b_{m, n}+b_{m+1, n}\right)^{*} \\
& \left.+V d_{m, n}\left(b_{m, n}+c_{m, n}+a_{m, n}+a_{m, n-1}\right)^{*}+\text { c.c. }\right\} .
\end{aligned}
$$

For the sake of simplicity and without loss of generality, we normalize the coupling parameter to $V=1$. Since the effective nonlinearity only depends on the product $\gamma P$, we may either consider the nonlinear coefficient $\gamma$ as a free parameter while fixing the mode amplitudes, so that the mode power is normalized to unity, or normalize the nonlinearity constant to $\gamma= \pm 1$ and consider the power $P>0$ and the sign of $\gamma$ as a free parameter. Depending on the context, both approaches are used alternatively in the numerics below. Moreover, we should stress that for the physical system studied here, the overall sign of the Hamiltonian is arbitrary, since changing the sign just amounts to a complex conjugation of the mode amplitudes or, equivalently, an inversion of the longitudinal coordinate $z$. We here chose a convention which allows one of the linear FBs to be interpreted as ground state, as in Ref. [33].

\section{B. Linear spectrum $(\gamma=0)$ and compact modes}

For infinite systems or finite systems with periodic boundary conditions on $m$ and $n$, stationary solutions can be expressed in a Bloch wave form: $\left\{a_{m, n}, b_{m, n}, c_{m, n}, d_{m, n}\right\} \sim$ $\{A, B, C, D\} e^{i\left(k_{x} m+k_{y} n\right)} e^{-i \beta z}$, where $\beta$ is the propagation constant, and $k_{x}, k_{y}$ are the components of the transversal 2D Bloch wave vector $\vec{k}$. In the absence of nonlinearity $(\gamma=0)$, we obtain the spectrum for linear solutions of this ODL, given by

$$
\beta_{1}=0, \quad \beta_{2}=2, \quad \beta_{3,4}=-1 \pm \sqrt{5+2 \cos k_{x}+2 \cos k_{y}} .
$$

Two bands $\left(\beta_{1}\right.$ and $\left.\beta_{2}\right)$ are $k$ independent and correspond to fully degenerated dispersion-less FBs [33]. The other two bands are dispersive and depend on $k_{x}, k_{y}$ wave vectors. The linear spectrum is shown in Fig. 2. The upper DB is connected to the upper $\mathrm{FB}$ at $\beta_{2}=2$, at the center of the Brillouin zone $\left(k_{x}=k_{y}=0\right)$. The lower $\mathrm{FB}\left(\beta_{1}=0\right)$ is also connected to the upper DB, but at the borders of the Brillouin zone $\left(\left|k_{x}\right|=\left|k_{y}\right|=\pi\right)$, as shown in Fig. 2 .

In a finite lattice with $M N$ unit cells and periodic boundary conditions, the degree of degeneracy of each FB is $M N$. Each of the FB eigenbases can be spanned by a corresponding set of compact, but not necessarily orthogonal, localized eigenstates (the FB compactons) [33], illustrated in Figs. 3(a) and 3(b). The reason for nonorthogonality is that each fundamental

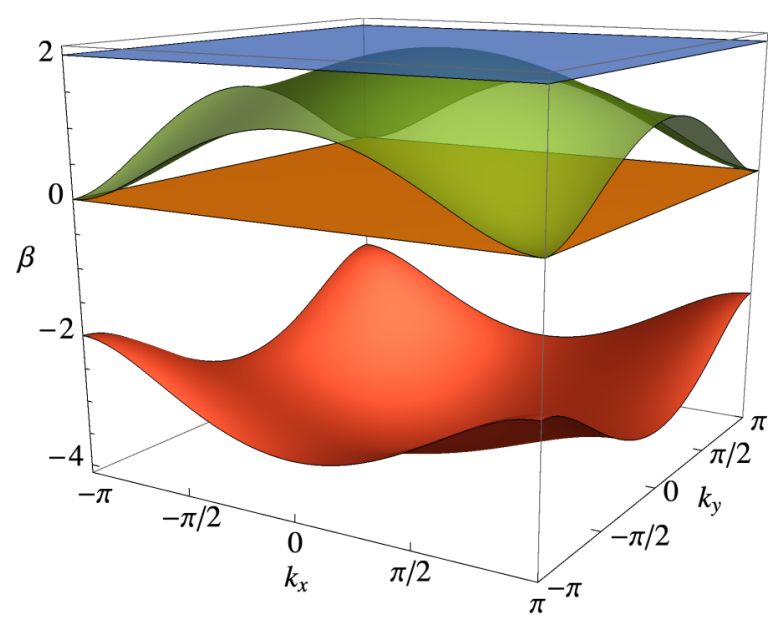

FIG. 2. Band-gap diagram (frequency spectrum) of the linear ODL. $\beta_{1}, \beta_{2}, \beta_{3}$ and $\beta_{4}$ are shown in orange (flat, $\beta=0$ ), blue (flat, $\beta=2$ ), green (upper dispersive), and red (lower dispersive), respectively.

compacton is an eight-site octagonal structure which is shared by four unit cells. In other words, the compactons belong to the class $U=4$ as defined in Ref. [4]. They originate from the geometrically ensured destructive interference effect outside of the compacton sites. Two types of fundamental homogeneous compactons, which are composed of eight sites each, having either dimeric $(++--++--)$ or monomeric $(+-+-+-+-)$ staggered phase structure are associated to the $\beta=0$ and $\beta=2 \mathrm{FBs}$, respectively. In the following we use the abbreviations $\mathrm{C} 1$ and $\mathrm{C} 2$, respectively, for these two types of compactons, as described in Fig. 3. Thus, compactons of types $\mathrm{C} 1$ and $\mathrm{C} 2$ are orthogonal to compactons of the same type only if they are not sharing a unit cell (i.e., if they are not localized on neighboring octagon rings), while compactons
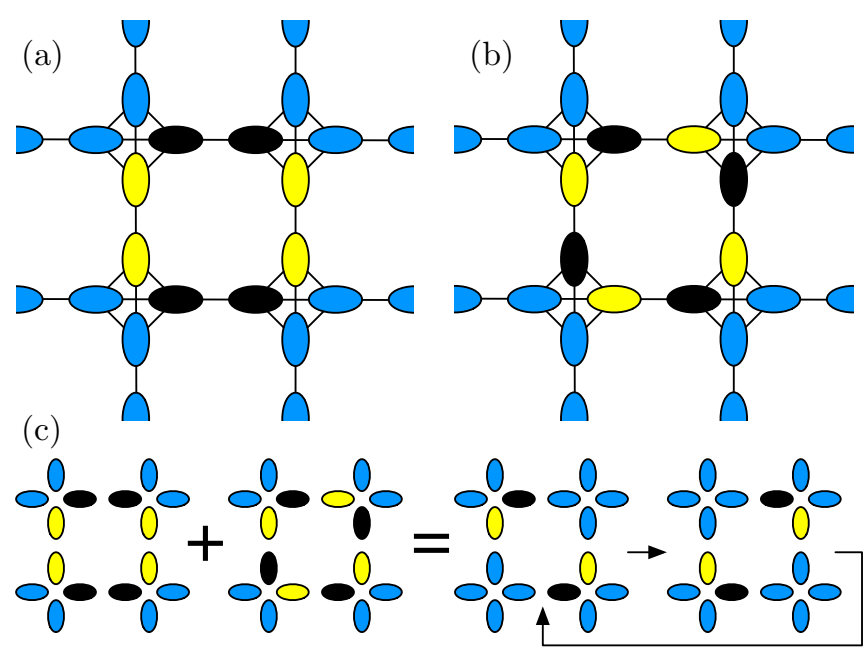

FIG. 3. Amplitude distribution of compacton solutions for (a) $\beta_{1}=0$ (C1) and (b) $\beta_{2}=2$ (C2). Yellow (black) corresponds to a positive (negative) amplitude, while light blue represents an empty site. (c) Linear combination of $\mathrm{C} 1$ and $\mathrm{C} 2$ shows an oscillating dynamics. 
of different types are always orthogonal since they belong to different bands.

We note also that any finite linear combination of compactons is also a compact solution of the linear system, which is stationary if all compactons are of the same type [22]. However, the linear combination of compactons will show amplitude oscillations, with frequency $\left|\beta_{2}-\beta_{1}\right|=2$, if overlapping compactons of different type are excited, as sketched in Fig. 3(c). This oscillation could be used as a precise "FB clock," which will show a precise frequency with a welldefined oscillating intensity pattern. In addition, this intensity oscillation could be used as a time-periodic mechanism for the application of energy at specific regions of a given lattice in order to, for example, excite a given interaction or reaction at well-defined positions.

\section{Numerical tools for analysis of stability and localization properties}

The stability properties of nonlinear stationary solutions are numerically studied by applying a standard linear stability analysis (LSA) [38] and checked by direct numerical simulations of model (1). Both of them are based on studying the evolution of initially small perturbations which are added to the stationary solutions. The LSA is applied by adding a small perturbation to the specific nonlinear mode whose stability properties are being investigated. Schematically, it can be written as follows:

$$
\Psi_{m, n}(z)=\psi_{m, n} \exp (-i \mu z)\left[1+\delta \psi_{m, n}(z)\right]
$$

where $\mu$ is the nonlinear propagation constant, and $\delta \psi_{m, n}$ a small perturbation.

By substituting Eq. (5) into the model equation and linearizing it with respect to small perturbations, the set of linear equations for the propagation of small perturbations can be derived. It can be solved as the eigenproblem of small perturbations, resulting in a set of eigenvalues (EVs) named instability EVs. They directly indicate the initial tendency of the mode evolution in the presence of small perturbations. Note that the LSA gives a sufficient but not necessary condition for the mode instability, and the real part of the EVs indicates the initial exponential growth rate of a small perturbation and the type of instability (pure real or complex, the latter resulting in oscillatory instability).

To characterize the instability of a given nonlinear solution, we define a stability index $G$ as the maximum real part among all eigenvalues, indicating the distance (time) where this mode will become unstable $(z \sim 1 / G)$. Therefore, in our analysis, stable solutions are represented by $G=0$, while unstable ones show a value $G>0$.

To confirm the LSA findings and obtain a more explicit presentation of the perturbed mode evolution, we perform direct numerical simulations of the model equation (1). Initially, the localized mode is injected into the system and its whole evolution is then governed by the effects modeled by the equations. We add initially a uniform random perturbation to the injected mode. The basic numerical procedure at this stage is the Runge-Kutta procedure of the sixth order. Here we confirm the long time mode stability by observing the time evolution of the participation number $R$, defined as

$$
R=\frac{P^{2}}{\sum_{m, n}\left(\left|a_{m, n}\right|^{4}+\left|b_{m, n}\right|^{4}+\left|c_{m, n}\right|^{4}+\left|d_{m, n}\right|^{4}\right)},
$$

and mode imbalance $I B$,

$$
I B=\frac{\sum_{i=n-1}^{n}\left(\left|a_{m-1, i}\right|^{2}+\left|b_{m-1, i}\right|^{2}+\left|c_{m-1, i}\right|^{2}+\left|d_{m-1, i}\right|^{2}\right)}{\sum_{i=n-1}^{n}\left(\left|a_{m, i}\right|^{2}+\left|b_{m, i}\right|^{2}+\left|c_{m, i}\right|^{2}+\left|d_{m, i}\right|^{2}\right)} .
$$

$R$ is a measure of the number of sites where the mode has its main localization (for a compact mode with homogeneous amplitude it gives exactly the number of sites on which the mode is localized), while $I B$ as defined above indicates the breaking of the internal mode symmetry in the $x$ direction for a mode with main localization on the four unit cells $(m-1, n-$ $1),(m-1, n),(m, n-1)$, and $(m, n)$ containing an octagon ring $(I B=1$ for a perfectly symmetric mode), with an obvious analogous definition for symmetry breaking in the $y$ direction.

\section{NONLINEAR LOCALIZED MODES}

\section{A. Instabilities of compact localized modes}

On-site nonlinearity allows to analytically continue single linear compact localized modes of type $\mathrm{C} 1$ and $\mathrm{C} 2$, initially originated at FBs, towards the gap or into the linear spectrum for increasing $|\gamma| P$. This results in continuous one-parameter families of compactons with preserved shape, in the sense that only the amplitude at each compacton site is equally modified for increasing power (nonlinearity strength), while the phase differences among sites are preserved. For these families of exact compactons, the nonlinear propagation constant $\mu$ is easily seen to be shifted from the linear $\beta$ by an amount exactly proportional to the power (nonlinearity strength), as

$$
\mu=\beta-\frac{\gamma P}{8} .
$$

In other words, the homogeneous compacton solutions continue to exist in the presence of an on-site cubic nonlinearity [5] with a participation number $R=8$, being affected by nonlinearity only via the amplitude of equally populated sites. However, these compact nonlinear localized modes are not necessarily stable and a stability analysis is required [28]. Compact localized modes are highly sensitive to the presence of any kind of perturbation which does not follow the internal symmetry of the compacton.

Since the modes are compact and the LSA (5) is performed in the frame rotating with $\mu$, the eigenvalue spectrum linearized around a single compacton mode in an infinite lattice will always contain purely imaginary eigenvalues given by the original linear spectrum (4), but shifted with $\frac{\gamma P}{8}$ according to Eq. (8). Moreover, due to the symplectic properties, the negative, as well as the complex conjugate of each eigenvalue, will also belong to the linearized spectrum, as will the eigenvalue zero corresponding to a pure phase rotation of the compacton. Finally, additional eigenvalues will appear due to localized eigenmodes, originating either from internal-mode oscillations or exponential instabilities inside the octagon ring, or from local oscillations at nonexcited sites neighboring the excited compacton, thereby inducing a nonlinearity-provoked 

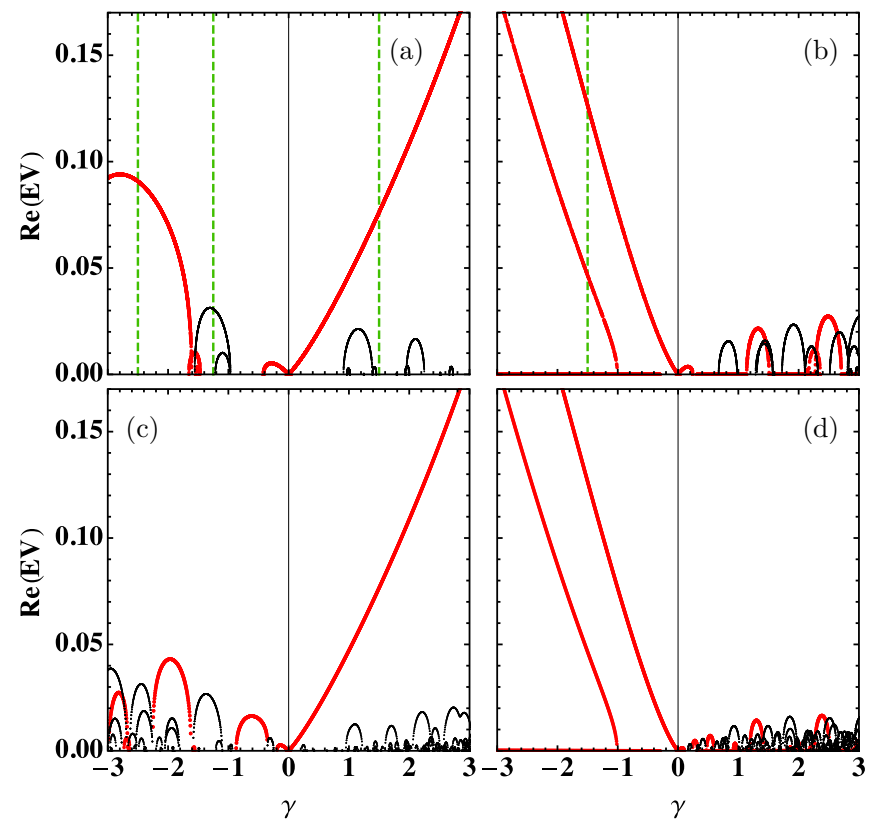

FIG. 4. Real parts of EVs for $\mathrm{C} 1$ and $\mathrm{C} 2$ compactons, for (a), (b) $7 \times 7$ and (c), (d) $15 \times 15$ ODLs, respectively. Pure real EVs are marked with red (thicker) symbols and real parts of complex EVs with black (thinner) symbols. Green dashed vertical lines in (a) and (b) correspond to simulations in Figs. 5 and 7, respectively.

interaction between the localized compact mode and the rest of the lattice. Resonances between eigenvalues of different origin typically yield instabilities. For a finite system, also the DBs will consist of discrete eigenvalues, yielding visible size effects for small system as shown below.

The LSA results for the nonlinear compact modes of the ODL with $49(7 \times 7)$ and $125(15 \times 15)$ unit cells are summarized in Fig. 4, for regimes of moderate focusing and defocusing nonlinearities (here and in the rest of this section, $P=1$ unless otherwise stated). Regimes of very weak and very strong nonlinearities are discussed separately below. Qualitatively the same feature, i.e., the presence of stability (weak instability) windows for both compactons with focusing nonlinearity and for the $\mathrm{C} 1$ compacton also for defocusing, is found for lattices having from $25(5 \times 5)$ to $400(20 \times 20)$ unit cells. We observe that an increment in the number of unit cells shrinks or closes the stability windows, with a slight reduction of the maximum values of real parts of EVs. The observed property is associated with finite-size effects $[39,40]$, typically appearing when internal-mode oscillations of the excited compacton resonate with frequencies from the linear dispersive bands. As the number of unit cells increases, the latter will approach a continuous spectrum, resulting in a remaining weak oscillatory instability with a smooth parameter dependence for the instability growth rate.

\section{Compacton C1 (dimeric phase structure)}

The propagation constant for this compacton is shifted by nonlinearity to $\mu=-\gamma P / 8$, and thus the linearized spectrum will contain imaginary eigenvalues corresponding to the original spectrum (2) but shifted downwards (upwards) for defocusing (focusing) nonlinearities, as well as their complex conjugates (sign reversed). Thus, for not too large defocusing nonlinearity (NL; $\gamma<0)$ the upper dispersive band will overlap at zero and generally yield instabilities corresponding to resonances between internal compacton modes and the dispersive band, and result in spreading and decay of the compacton in larger lattices. For smaller lattices, stable windows may appear as discussed above, due to the discreteness of the dispersive band.

On the other hand, for focusing NL $(\gamma>0)$ the linear spectrum will be shifted upwards, and since the upper DB moves away from $\beta=0$ and the lower DB is gapped, no resonances with dispersive modes should be expected until the nonlinearity is large enough to overcome the gap to the lower DB. Still this compacton is found to be unstable with purely real eigenvalue for any $\gamma P>0$. The explanation for this instability is connected to the internal phase dynamics of the compact octagon ring, containing neighboring pairs of excited sites with identical phases. As discussed, e.g., in Refs. [41,42], such phase interactions generically yield instabilities for focusing and large enough nonlinearities; in the terminology of Refs. [41,42], the $\mathrm{C} 1$ octagon ring corresponds to a finite part of a standing wave of "type E" with wave vector $Q=\pi / 2$, consisting of a periodic repetition of the pattern $(++--)$. For the pure octagon ring, this instability appears for $\gamma P / V \gtrsim 5.6$ (the analogous instability for the fundamental four-site pattern was seen in Ref. [43]; the threshold decreases with increasing ring size and goes to zero in the limit of an infinite chain [42]). However, this amplitude pattern also has an additional neutral eigenvalue, from the degeneracy corresponding to an arbitrary rotation of the relative phase between odd and even sites (thus termed " $\pi-\pi$ states" in Ref. [44] and "phase states" in Ref. [45]). The presence of the surrounding zero-amplitude sites in the ODL breaks this degeneracy and turns the neutral mode into an unstable eigenmode with a purely real eigenvalue, as seen in Figs. 4(a) and $4(\mathrm{c})$.

In the limit of very strong nonlinearities $(|\gamma| P / V \rightarrow \infty$, equivalent to the weak-coupling, "anticontinuous" limit $V \rightarrow$ 0 ), the linear band structure becomes irrelevant and only phase interactions within the compacton ring determine its stability. Considering in this limit only the octagon as a onedimensional chain, the defocusing case becomes equivalent to the focusing case by sign reversals of the amplitude at every second site ("staggering transformation"), and since this sign reversal results in an octagon with the same phase structure (only shifted one lattice site), the above argument [41,42] proves instability in the large-nonlinearity regime also for the defocusing case.

The above conclusions from the LSA are illustrated by direct numerical simulations in Figs. 5 and 6 (left part). In Fig. 5 the evolution of the participation number (R) (6) and the imbalance (IB) (7) is plotted for certain values of the NL parameter, which belong to different instability regions in the ODL with 49 cells. These values are marked by vertical dashed lines on the corresponding EV diagram in Fig. 4(a). As can be seen, for negative nonlinearity there is an approximately simultaneous increase in $\mathrm{R}$ and decrease in IB, indicating an instability that simultaneously breaks the symmetry of the compacton and causes it to spread in the 

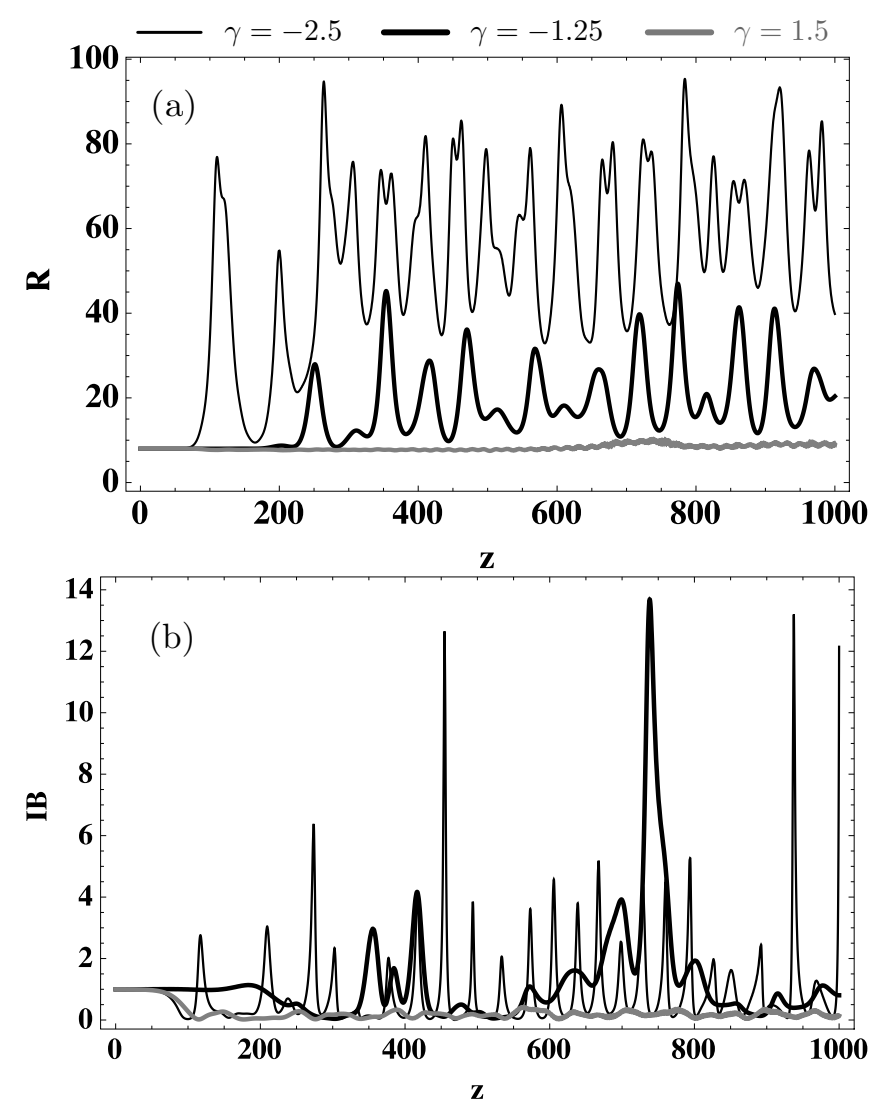

FIG. 5. (a) Participation number and (b) imbalance for $\mathrm{C} 1$ in ODL with $49(7 \times 7)$ cells. The selected values of $\gamma$ are shown by vertical lines in the EV diagram, Fig. 4(a).

lattice, consistent with the LSA results of internal compacton modes resonating with DB.

On the other hand, for positive $\gamma$ there is a rapid decrease of IB to very small numbers, indicating a strong symmetry breaking of the compacton, and only weak oscillations in $\mathrm{R}$ developing on larger time scales. Also this is consistent with LSA results of an unstable internal mode without direct resonance with the linear DBs, allowing for a well-localized (although nonstationary and noncompact) mode to survive for long distances.

Another illustration of the compacton stability properties is shown in Fig. 6, where we present the mode amplitude distribution over the cells at the initial time, and a certain time instant during the propagation through the relatively small lattice with $7 \times 7$ unit cells. The left column corresponds to the case with an initially launched $\mathrm{C} 1$ solution in the central part of the lattice. The values of the NL parameter are $\gamma=-1,1$, and 2 , respectively. The first plot illustrates the behavior of the nonlinear compact localized mode $(\gamma=-1)$ (note the logarithmic scale) which is on the border of the stability window in Fig. 4(a) caused by the finite size of the system. There the mode appears as essentially stable over long time scales; only weak background fluctuations have been detected. The other two cases, $\gamma=1$ and $\gamma=2$, correspond to the unstable mode with a real eigenvalue, associated with an internal symmetry breaking of the octagonal compacton
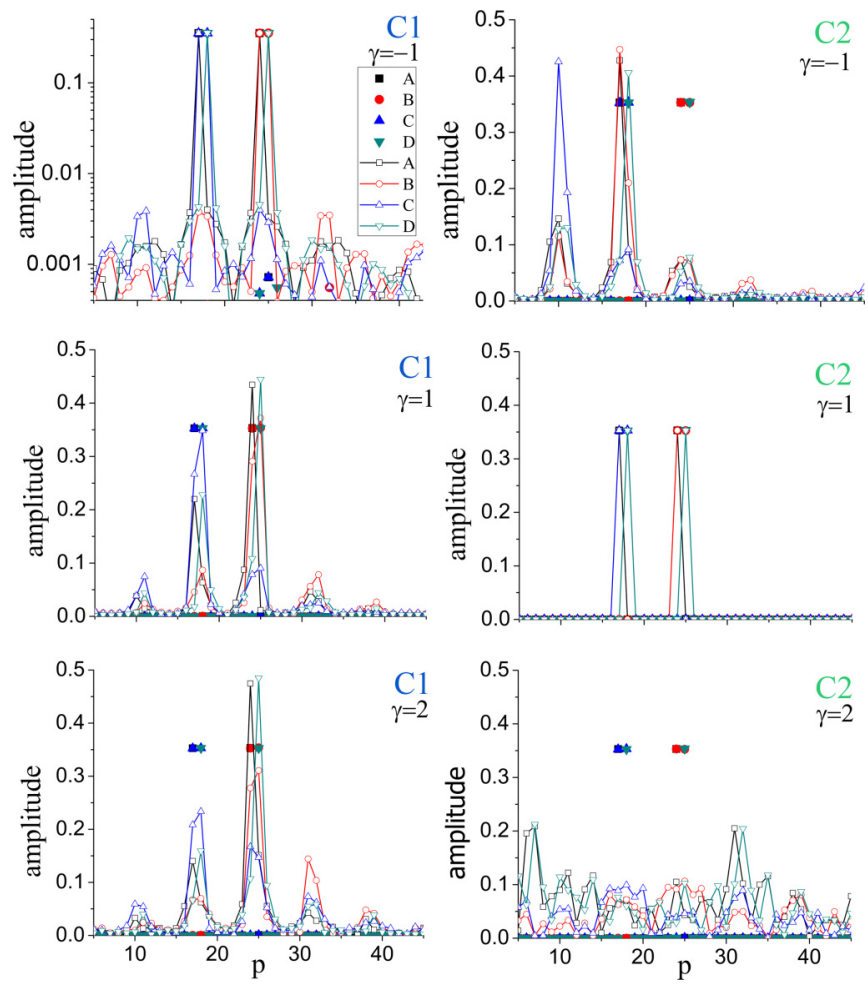

FIG. 6. Amplitude profiles at sites $a, b, c$, and $d$ during the evolution. Solid symbols denote the initial stage, while the open symbols and solid lines are time snapshots during the evolution. The selected are values of the NL parameter from characteristic instability regions for $\mathrm{C} 1$ and $\mathrm{C} 2$. Abbreviations in boxes $A, B, C$, and $D$ denote the corresponding sites, while $p=m+(n-1) N$, where $m, n=1, \ldots, N(N=7)$ indexes cells (ODL of $7 \times 7=49$ cells is considered).

together with a leaking of energy mainly towards the neighboring cells in the lattice.

\section{Compacton C2 (monomeric phase structure)}

Here, $\mu=2-\gamma P / 8$, and thus the original spectrum (2) appears in the imaginary part of the linearized spectrum as $\operatorname{Im}(E V)=\beta-2+\gamma P / 8$. Thus, for not too large focusing NL $(\gamma>0)$ the upper dispersive band will overlap at zero, and instabilities corresponding to resonances between internal compacton modes and the dispersive band appear, resulting in spreading and decay of the compacton in larger lattices and stable windows for smaller systems, similarly as for the $\mathrm{C} 1$ compacton with defocusing nonlinearity.

In the case with defocusing $(\gamma<0) \mathrm{NL}$, the propagation constant $\mu$ enters the semi-infinite gap (SIG) region above $\beta=2$, and thus no resonances with extended modes appear in the LSA for the C2 compacton, for any defocusing nonlinearity. Thus, due to the similarity of this part of the band structure with that of the kagome lattice, one might here a priori expect similar stability properties also for the corresponding compact mode. The compacton in the kagome lattice remains as a stable ground state (minimizer of Hamiltonian for fixed norm) of the system for a regime of weak nonlinearity, but destabilizes due to internal symmetry breaking with respect to a single-site peaked mode for larger 


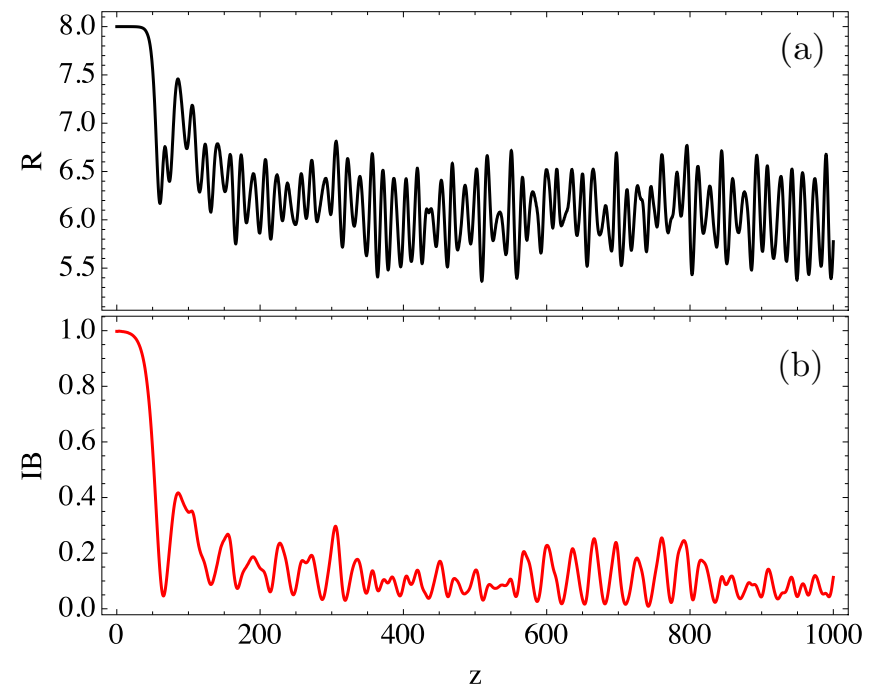

FIG. 7. (a) Participation number and (b) imbalance for $\mathrm{C} 2$ in ODL with $49(7 \times 7)$ cells. The selected value of $\gamma$ is shown by a dashed vertical line in the EV diagram, Fig. 4(b).

nonlinearities [27]. However, the scenario for the ODL C2 compacton turns out to be different. In fact, for a sufficiently large system size, it destabilizes with a purely real eigenvalue for any arbitrarily weak defocusing nonlinearity [Figs. 4(b) and 4(d)]. The reason for this destabilization is explored in more detail in the following section, where we find that as soon as the nonlinearity becomes nonzero, the ground state of a sufficiently large system is not a single $\mathrm{C} 2$ compacton but a nontrivial linear combination of $\mathrm{C} 2$ compactons positioned at different octagon rings.

Examples from direct numerical simulations of the outcome of instabilities of the $\mathrm{C} 2$ compacton are shown in Figs. 7 and 6 (right part). For the defocusing nonlinearity, we see from Fig. 7 that $R$ is decreasing, indicating a transition into a more localized structure, and that $I B$ decreases to small numbers, indicating a strong symmetry breaking of the octagon ring. Thus, this is consistent with a generic self-trapping mechanism governing the localization properties, with the injected energy being redistributed into new structures with nonzero but decaying tails (see example in upper right of Fig. 6). For positive $\gamma$, the scenario is qualitatively similar as for the $\mathrm{C} 1$ compacton with defocusing nonlinearity: inside the stable windows for small systems the compacton remains largely unaffected (see example for $\gamma=1$ in middle right of Fig. 6), while in the regimes of resonance with the DB (example for $\gamma=2$ in lower right of Fig. 6) a strong spreading in the lattice is seen.

In the large-nonlinearity regime of the $\mathrm{C} 2$ compacton, $|\gamma| P / V \gg 1$, the essential dynamics is governed by the internal dynamics of the excited octagon ring, and the rest of the lattice can be considered as a weak perturbation. In the defocusing case $(\gamma / V<0)$, the staggered-phase structure in the octagon then always breaks up due to modulational instability, and spreading to the rest of the lattice only appears to higher order in the small parameter $V /|\gamma| P$. On the other hand, for focusing nonlinearity $(\gamma / V>0)$ the staggeredphase structure in itself is stable (ground state of the single

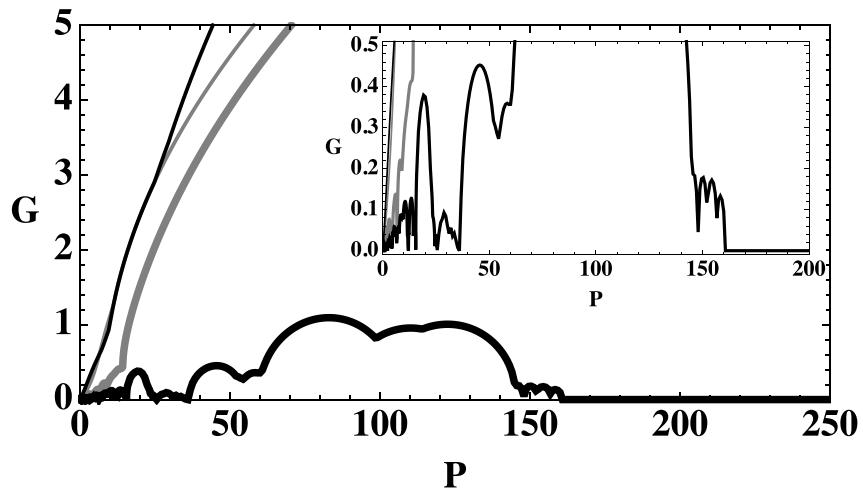

FIG. 8. Maximum instability growth rate $G$ vs power $P$ for compactons in the ODL with $64(8 \times 8)$ cells. Thick gray line, $\mathrm{C} 1$ compacton $\gamma=-1$; thin gray line, $\mathrm{C} 1$ compacton, $\gamma=1$; thin black line, $\mathrm{C} 2$ compacton, $\gamma=-1$; thick black line, $\mathrm{C} 2$ compacton $\gamma=1$.

octagon ring), and instabilities may only appear at some small but nonzero threshold of $V / \gamma P$ through coupling to zeroamplitude lattice sites outside the ring. The stabilization of the C2 compacton at large values of $\gamma P / V \gg 1$ is also confirmed numerically (see Fig. 8 where also a comparison with the most unstable eigenvalues for the $\mathrm{C} 1$ compacton is shown). The stable regime for the focusing $\mathrm{C} 2$ compacton is thus seen to be approximately $\gamma P / V \gtrsim 160$ (for a large enough system where finite-size effects can be neglected). The mechanism for destabilization is analogous to that of, e.g., discrete dark solitons [40], as both appear due to the coupling of a staggered mode to additional zero-amplitude sites. However, the power necessary for stabilization is considerably larger for the $\mathrm{C} 2$ compacton, as there is more than one zero-amplitude site connecting to the same pair of antiphased compacton sites [see Fig. 3(b)].

\section{B. Noncompact localized modes in the semi-infinite gap $\boldsymbol{\mu}>\mathbf{2}$}

\section{Numerical continuation versus power}

Here, we investigate a variety of localized patterns existing in the SIG above the linear FB at $\beta=2$ in the presence of a defocusing nonlinearity. For the numerics in this section, we put $\gamma=-1, V=1$ and consider the power $P$ as a nonlinearity parameter.

A summarizing picture of the considered mode families is illustrated by their amplitude profiles in the high-power (anticontinuous) limit and the power vs $\mu$ dependencies (see Fig. 9). These curves are obtained by numerical continuation of the shown patterns in the direction of decreasing $\mu$ by implementing a multidimensional Newton-Raphson method. Except for the $\mathrm{C} 2$ compacton ring mode (light gray dashed line), whose exact (linear) $P(\mu)$ dependence follows from Eq. (8), the power depends nontrivially on the propagation constant, reflecting the fact that these modes generally show exponentially decaying tails and in that sense are "standard" discrete solitons.

As expected, several of these modes disappear in bifurcations with other modes (not shown in the picture) before reaching the linear band limit $\beta=2$, but we identify four qualitatively different modes (with different symmetry 

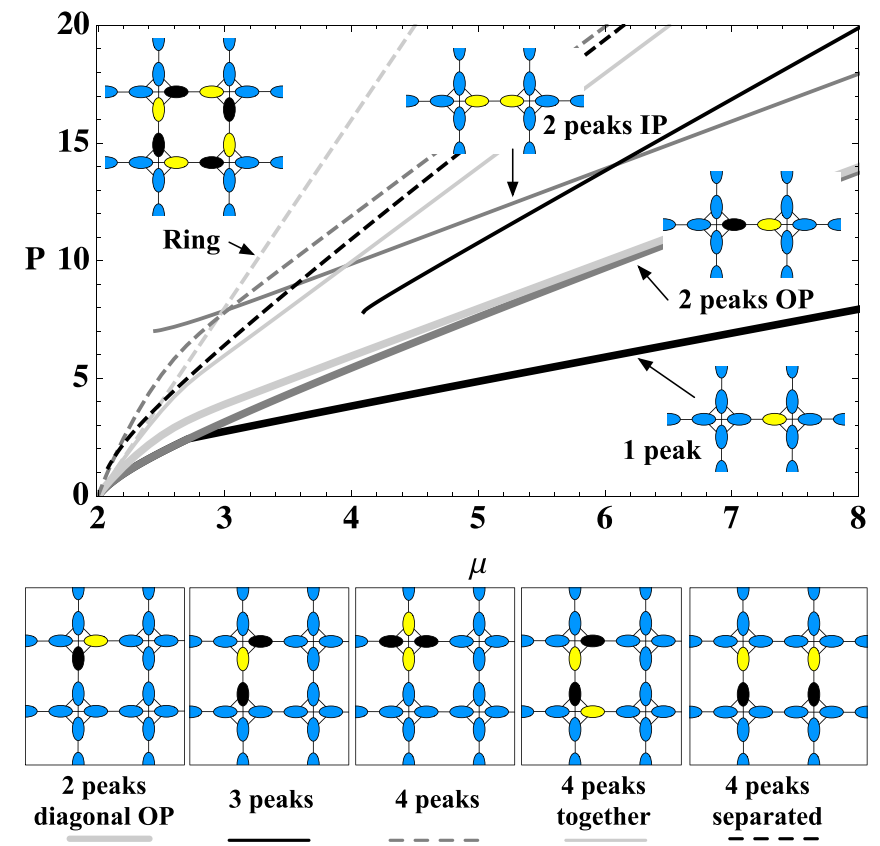

FIG. 9. $P$ vs $\mu$ diagram of different families of nonlinear localized modes for $\gamma=-1$. Representative mode profiles, in the large-power (anticontinuous) limit, are shown in insets and indicate their respective line type. For all modes, yellow (light gray) and black correspond to a positive and negative amplitude, respectively, while blue (gray) represents an empty site. (Abbreviations IP and OP stand for in-phase and out-of-phase, respectively.)

properties) that may be continued all the way to the zeropower limit at $\beta=2$. This is more clearly seen in Fig. 10, where the participation number $(R)$, Hamiltonian $(H)$, and maximum instability growth rate $(G)$ are plotted vs mode power $(P)$. For large nonlinearities, a single-peaked solution (black curves) always constitutes the stable ground state, as for the ordinary square DNLS lattice. However, the singlepeaked modes only exist for $P \gtrsim 2.4$, where they bifurcate through a pitchfork bifurcation from a solution (dark gray solid line) with two out-of-phase (OP) peaks placed horizontally (or, equivalently, vertically due to fourfold rotation symmetry of the ODL). For all weaker nonlinearities, this family of two-peaked localized modes will remain as the stable ground state, and as is seen from Fig. 10(a), it will also be the most localized state in the sense of having the smallest participation number. Note in particular that the two-peaked mode always has smaller $H$, and smaller $R$, than the $\mathrm{C} 2$ compacton ring mode (light gray dashed line), and thus the latter will always be prone to symmetry-breaking instabilities as seen in the previous section. The other two solutions that continue to the linear limit are a similar antiphased two-peak solution (thick light gray solid line) but with the peaks placed "diagonally" (i.e., on two neighboring sites in the same unit cell), and a solution with all four sites in a unit cell excited in antiphase (dashed gray line). However, these modes are generally unstable [see Fig. 10(c)], although it is interesting to note that also the diagonal two-peaked mode has a smaller value of $H$ than the $\mathrm{C} 2$ compacton in the small-power regime [inset in Fig. 10(b)].
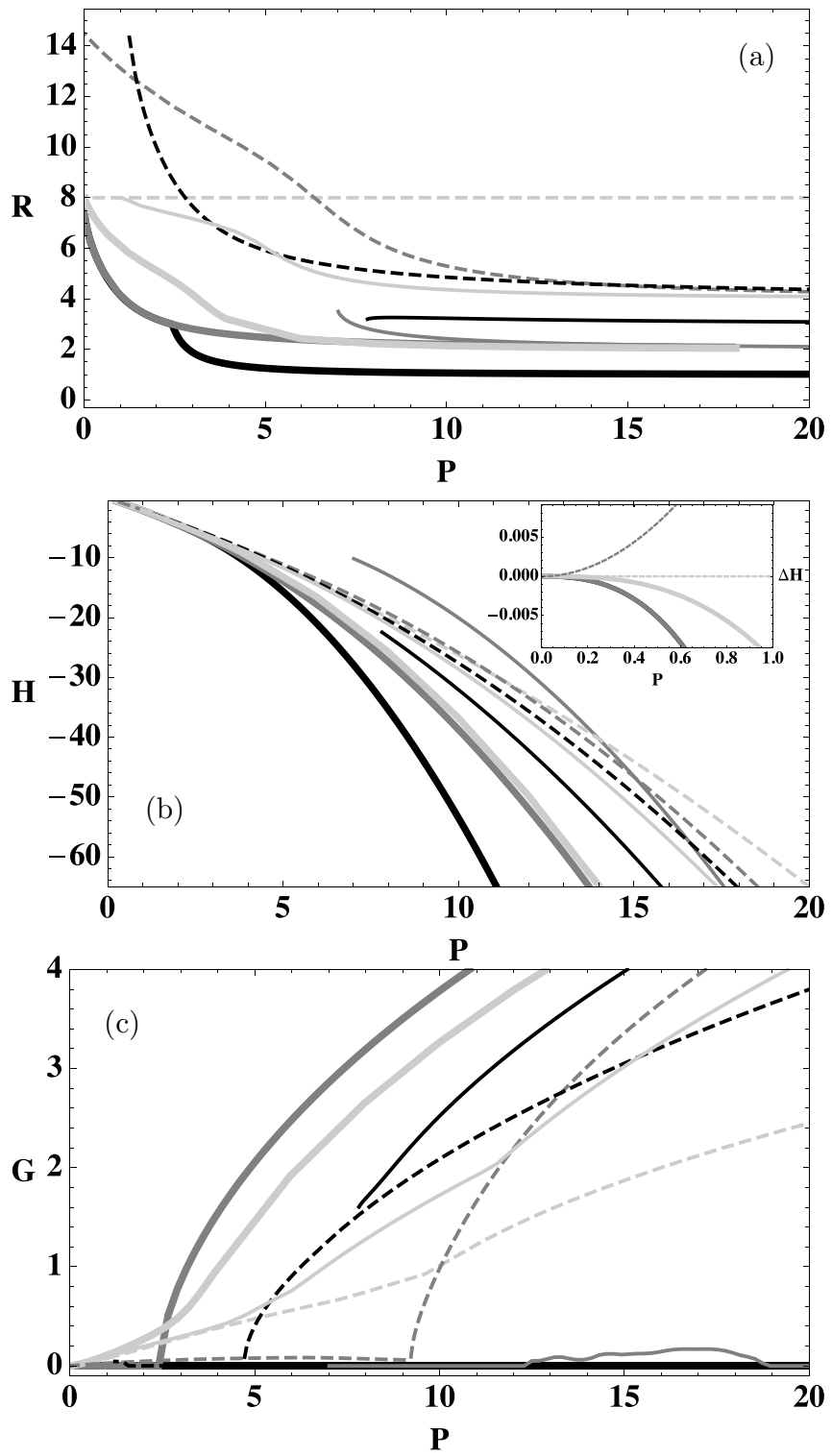

FIG. 10. (a) Participation number $R$, (b) Hamiltonian $H$, and (c) instability rate $G$ vs power $P$, for all modes shown in Fig. 9 and using the same line types. Inset in (b) shows the difference $\Delta H$, compared to $H$ for the $\mathrm{C} 2$ compacton $(\Delta H=0)$, for solutions which continue to $\beta=2$.

Note also from Fig. 10 that, although the ground state is unique (modulo lattice translations), several other linearly stable stationary modes may exist for the same power in regimes of larger nonlinearities. Thus, for suitably chosen initial conditions also these modes should be expected to survive for long propagation distances under realistic experimental conditions.

\section{Size dependence of ground state for weak nonlinearity}

In the linear limit $(\gamma=0)$, any linear combination of $\mathrm{C} 2$ compacton ring modes is an exact stationary solution with Hamiltonian $H=-2 P$, and thus all such solutions are part of a highly degenerated ground state. However, not all these solutions remain stationary as nonlinearity is turned on. In 
general only those linear combinations that extremalize $H$ within a given symmetry class are expected to survive. Since it follows directly from the definition of the participation number that $H$ can be expressed as [46]

$$
H=-\mu P-\frac{\gamma P^{2}}{2 R}
$$

the condition to extremalize $H$ in the linear limit $\mu=2$ becomes equivalent to extremalize $R$ for fixed $P$, and in particular we should expect the ground state at weak nonlinearity to be the linear combination which minimizes the participation number, i.e., the most strongly localized mode.

Comparing with the analogous scenario for the kagome lattice [27], one might a priori expect that the two primary candidates for the weak-nonlinearity ground state would be the single-ring mode with $R=8$ and a mode with two neighboring (horizontal or vertical) rings excited in antiphase, so to create a structure with two main antiphased peaks in the center, as indicated in Fig. 9 (dark gray solid line). However, a straightforward calculation for the latter mode yields $R=$ $100 / 11=9.09>8$, so a pure two-ring mode would have larger $H$ than the single ring for weak nonlinearity, and thus it could not be the ground state. Even if we attach two additional rings to constitute a linear segment of four rings, a straightforward optimization procedure yields a minimal $R \approx$ 8.70 for such a linear combination, and thus the single-ring mode would still constitute the ground state for a small system consisting only of these four rings. On the other hand, it is clear from the numerical continuation in Fig. 10 that for a large enough system, the ground state is not a single ring but a two-peaked mode with $R<8$ as $P \rightarrow 0$. Thus, there should be a critical system size where the weakly nonlinear ground state changes from being a single compact ring to a nontrivial linear combination of ring modes.

In order to determine this critical system size, guided by our numerical results, we make an ansatz consisting of a linear combination of 14 compacton rings as follows:

$$
\begin{aligned}
\psi= & x\left(R_{0,0}-R_{0,1}\right)+y\left(R_{0,-1}-R_{0,2}\right)+z\left(R_{0,-2}-R_{0,3}\right) \\
& +u\left(R_{1,0}-R_{1,1}+R_{-1,0}-R_{-1,1}\right) \\
& +v\left(R_{1,-1}-R_{1,2}+R_{-1,-1}-R_{-1,2}\right) .
\end{aligned}
$$

Here, $R_{i, j}$ denotes a C2 compacton ring mode with unit amplitude at lattice position $(i, j)$, and $x, y, z, u$, and $v$ are positive coefficients to be determined from the condition to minimize $R$. The structure is sketched in Fig. 11, using a scaled profile in order to visualize this composed profile.

As shown in the Appendix, this minimizing solution indeed has a participation number $R \approx 7.79<8$, and so it should constitute the weak-nonlinearity ground state for a system consisting only of these 14 rings. Moreover, also a slightly smaller system with 12 rings (4 central rings in each row in Fig. 11) has $R<8$, but for any system containing less than 12 rings the minimum value of $R$ is larger than 8 (see the Appendix). Thus, the weak-nonlinearity ground state should be a two-peaked mode for all systems containing 12 rings or more, and a single-ring mode for smaller systems.

To see how this transition of the ground state reflects itself in the dynamics, we first illustrate in Fig. 12 the stability index versus power for the single $\mathrm{C} 2$ compacton put in

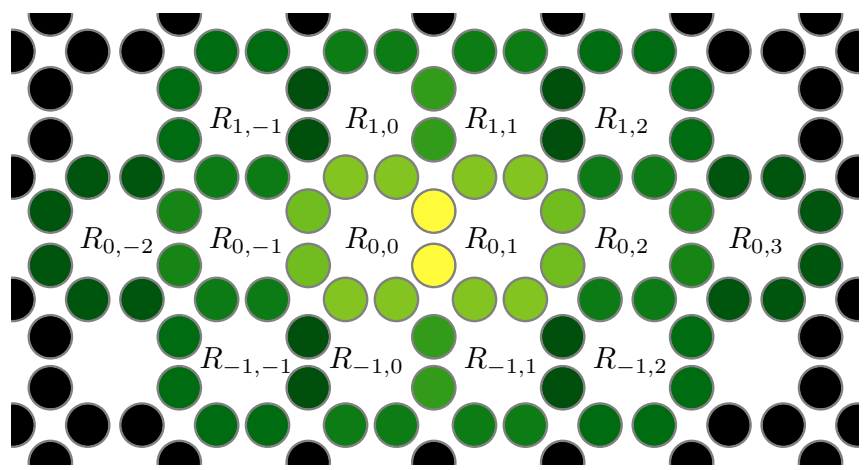

FIG. 11. $\sqrt{|\psi|}$ of the numerically obtained ground state in the weak-power limit $\left(P \approx 5 \times 10^{-6}\right)$. The 14 rings used in the ansatz (10) are 6 in the central row $(i=0,-2 \leqslant j \leqslant 3)$ and 4 in each of the top and bottom rows $(i= \pm 1,-1 \leqslant j \leqslant 2)$. Color code indicates an increasing amplitude going from black to yellow.

small systems consisting only of the indicated number of complete rings (i.e., not complete four-site unit cells) with fixed boundary conditions. As can be seen, for the four-ring system where the single compacton is the weak-nonlinearity ground state, it is, as expected, stable in a considerable regime of small power, $P \lesssim 0.55$. When the system size is increased to 12,14 , and 18 rings, where the compacton is no longer a ground state, the instability threshold decreases drastically. However, it should be noted that, even for these system sizes, there is a tiny regime of stability for small power, decreasing with system size. Thus, even if the compacton is not the ground state for these systems, it is apparently (at least linearly) metastable. As another check, we also performed direct numerical simulations for small systems consisting of complete four-site unit cells, using initial conditions close to those obtained by minimizing $R$ for the ansatz (10), for the largest number of complete rings contained in the system (see the Appendix for exact numbers). Thus, we confirmed that for system sizes $(3 \times 2),(5 \times 2)$, and $(7 \times 2)$ unit cells the average participation number remains larger than 8 , while for systems with $(5 \times 4)$ and $(7 \times 4)$ unit cells it remains smaller than 8 , for large times, weak nonlinearity, and small random perturbation. Thus, the critical system size of 12 rings for transition of the weak-nonlinearity ground state from

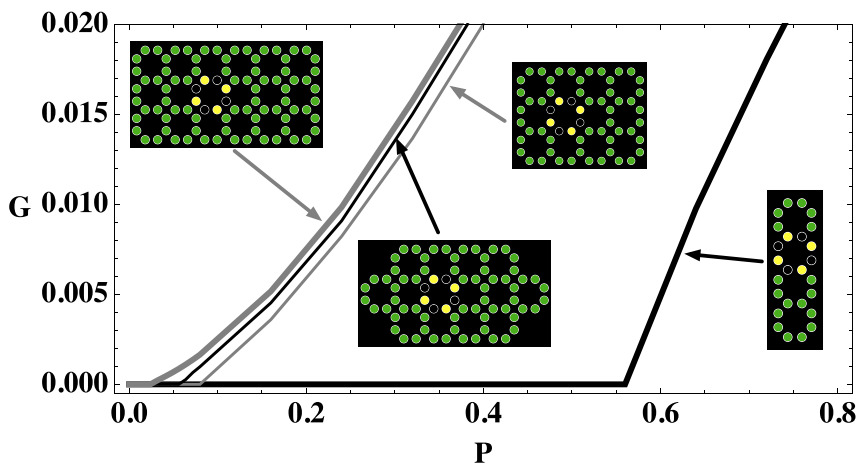

FIG. 12. Stability index $G$ vs power $P$ for a single $\mathrm{C} 2$ compacton put into small systems consisting only of the sites indicated in the respective insets ( $\gamma=-1, V=1$, fixed boundary conditions). 

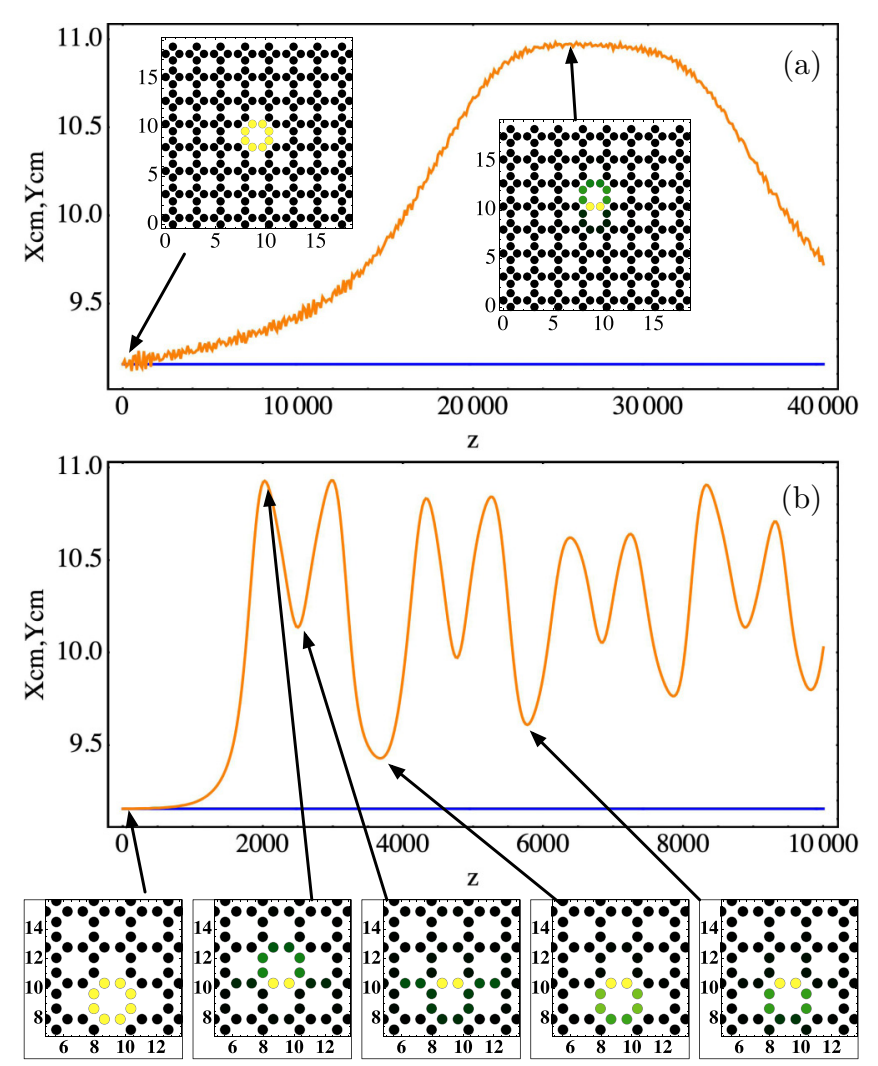

FIG. 13. Dynamical examples of small-power C2 compactons for $\gamma=-1, V=1$. We kick them in the vertical direction only $\left(k_{x}=0\right)$ for $\left\{k_{y}, P\right\}$ : (a) $\{-0.1,0.005\}$ and (b) $\{-0.001,0.1\}$. Main figures show the vertical $Y \mathrm{~cm}$ (orange) and horizontal $X \mathrm{~cm}$ (blue) center of mass vs $z$. Insets show intensity distributions at distances indicated by arrows. Fixed boundary conditions were used on a lattice of 256 sites.

single-ring to multiring mode is seen even if the system is not a pure 12 -ring system but a $(5 \times 4)$ unit cell system.

As we increase the system size, more rings are added to the linear combination (10), but with coefficients decreasing with distance to the center. Thus, we find numerically, e.g., that the participation number for the ground state in the small-power limit decreases to $R \approx 7.48,7.37$, and 7.34 for a system with $256(8 \times 8), 576(12 \times 12)$, and $784(14 \times 14)$ sites (unit cells), respectively. But the qualitative nature of the weaknonlinearity ground state with two main antiphased central peaks remains the same for all systems containing 12 rings or more.

\section{Numerical investigation of mobility}

Owing to the similarity of the band structure around $\beta=2$ to that of the kagome lattice, where good mobility of strongly localized modes was found in the vicinity of the FB-SIG border [27], additional effort was done to identify possible mobile modes also for the ODL in this regime. Two typical examples are illustrated in Fig. 13, where an initial C2 compacton $\left(R_{m, n}\right)$ in the vicinity of the lower SIG border has been transversely perturbed by a phase kick: $\psi_{m, n}(0)=R_{m, n} \exp [i(\vec{k} \cdot \vec{r})]$, where $\vec{r}$ defines the positions of compacton lattice sites across the lattice and $\vec{k}=\left\{k_{x}, k_{y}\right\}$, according to standard procedure to study mobility (see, e.g., Ref. [27]). As can be seen, no proper mobility appears, but rather the mode ends up oscillating back and forward in the effective Peierls-Nabarro potential well [47-49] created around the neighboring two-peaked ground state. A larger phase gradient does not facilitate for the mode to "jump over" the Peierls-Nabarro barrier to the next ring position, but instead excites more internal modes and radiation. Heuristically, we may interpret this as a consequence of the stable weak-nonlinearity ground state being the nontrivial linear combination of many ring modes. It is in contrast to the simple two-ring mode playing the corresponding part for the mobility in the kagome lattice. As the initial ODL ring mode translates towards the stable two-peaked mode, many additional ring modes get excited, which may not easily be reassembled again to a pure single, translated ring mode.

We also investigated the possibility of mobility of a ring mode in diagonal directions, which could be anticipated as that there exist stationary diagonal two-peak as well as fourpeak stationary solutions (thick light gray solid and dashed gray lines, respectively, in Figs. 9 and 10) that could constitute intermediate paths in a diagonal movement. However, the outcome turns out to be similar with those in axial movement: the mode does not translate but initially oscillates in the diagonal direction, and as an additional symmetry-breaking instability develops, its center of mass begins to perform independent oscillations in the horizontal and vertical directions (figure not shown). Thus, it appears that there is no translational mobility in any direction for the localized modes close to the upper flat band.

\section{CONCLUSIONS}

The octagonal-diamond lattice is known to have some interesting theoretical properties, in particular for the case where the diagonal coupling inside unit cells is fine tuned to be identical to the nearest-neighbor couplings. This results in a linear dispersion relation with two exactly flat bands and two orthogonal flat-band octagonal compactons with identical amplitude distributions but different phase structures. We proposed a way to experimentally realize such a lattice with elliptical waveguides and analyzed the properties of its localized modes in the linear and Kerr nonlinear regimes. We found that FB localized states exist as nonlinear compact solutions in the presence of nonlinearity. However, for large systems they typically become unstable if we switch on the nonlinearity, excepting the $\mathrm{C} 2$ compacton at high power for focusing nonlinearity. The instability can, however, be weak in certain areas of the parameter space $(\gamma, \mu, P)$ and although strict compactness will be dynamically destroyed, strong localization may still persist.

Therefore, from the point of view of the applications, the ODL could be of interest mostly in the use of its linear properties. With two orthogonal flat bands, a FB oscillation could be implemented to effectively disseminate a controlled amount of energy on a given lattice region, and with a very well-defined frequency. In addition, as this lattice possesses a full set of linear compact $\mathrm{C} 1$ and $\mathrm{C} 2$ states, the amount of possible combinations of compact states goes as $2^{\text {Ring }}$ (in- and out-of-phase excitations) [6,50]. This codification could be increased as well by exciting simultaneously both FBs, which 
would generate an oscillating pattern [Fig. 3(c)] and give an extra degree of freedom to increase the possible incoherent superpositions on different lattice regions. This is certainly an important goal nowadays in optical communication systems, where different forms of codification are required for efficiently encrypting a huge amount of digital data.

The nonlinear ODL also exhibits intriguing finite-size effects which to our knowledge has not been seen in earlier studied flat-band models. We showed that the weak-nonlinearity ground state in the defocusing regime switches from being a single compacton to a nontrivial combination of compactons at a critical system size consisting of 12 compacton rings. Another interesting effect for defocusing nonlinearity is the existence of a critical nonlinearity strength (or, equivalently, critical power) where the ground state (for large systems) switches from being double peaked to single peaked, through a symmetry-breaking pitchfork bifurcation.

The diversity of localized modes induced by the nonlinearity in the FB ODL also directs to investigations of fundamental processes responsible for dynamical pattern formation in such geometries, which is an important topic for future research.

\section{ACKNOWLEDGMENTS}

This work was supported in part by Programa ICM Millennium Institute for Research in Optics (MIRO), FONDECYT Grant No. 1191205 and the Ministry of Education, Science and Technological Development of Republic of Serbia (Project III45010).

\section{APPENDIX: EXPLICIT FORMULAS FOR WEAK-NONLINEARITY GROUND STATE IN SMALL SYSTEMS}

With the 14-ring ansatz (10) for a ground state with two antiphased central peaks, minimizing $R$ as defined in Eq. (6) becomes equivalent to finding the maximum of the function $f(x, y, z, u, v)=32 x^{4}+4(x-y)^{4}+4(y-z)^{4}+12 z^{4}+$ $8(x-u)^{4}+8(y-v)^{4}+72 u^{4}+8(u-v)^{4}+16 v^{4} \quad$ under the condition that $g(x, y, z, u, v)=8 x^{2}+4(x-y)^{2}+4(y-$ $z)^{2}+12 z^{2}+8(x-u)^{2}+8(y-v)^{2}+24 u^{2}+8(u-v)^{2}+$ $16 v^{2}=1$. With help of MATHEMATICA, the following solution is obtained: $x \approx 0.242999, y \approx 0.0693781, z \approx 0.0170956$, $u \approx 0.0427998, \quad v \approx 0.0279854$, which minimizes the participation number to $R \approx 7.79016<8$, and thus yields the ground state for the 14-ring system.

From the above expressions for $f$ and $g$ for the 14ring ansatz, we may directly obtain results also for smaller systems. Putting $z=u=v=0$ we obtain the 4-ring mode with minimum $R \approx 8.70176>8, u=v=0$ yields the 6-ring mode with minimum $R \approx 8.66596>8, z=v=0$ yields the 8-ring mode with minimum $R \approx 8.12148>8, v=0$ yields the 10-ring mode with minimum $R \approx 8.08559>8$, and $z=0$ yields the 12-ring mode with minimum $R \approx 7.85557<8$. Thus, we conclude that a two-peaked mode should constitute the weak-nonlinearity ground state for systems consisting of 12 rings or more, while the single-ring mode should be the ground state for systems containing less than 12 rings. The coefficients for the 12-ring mode are $x \approx 0.242178, y \approx$ $0.0631006, u \approx 0.042197, v \approx 0.0262899$.
[1] C. Kittel, Introduction to Solid State Physics, 7th ed. (Wiley, New York, 1996).

[2] D. Leykam, A. Andreanov, and S. Flach, Adv. Phys. X 3, 1 (2018).

[3] D. Leykam and S. Flach, APL Photonics 3, 070901 (2018).

[4] S. Flach, D. Leykam, J. D. Bodyfelt, P. Matthies, and A. S. Desyatnikov, Europhys. Lett. 105, 30001 (2014).

[5] L. Morales-Inostroza and R. A. Vicencio, Phys. Rev. A 94, 043831 (2016).

[6] R. A. Vicencio, C. Cantillano, L. Morales-Inostroza, B. Real, C. Mejía-Cortés, S. Weimann, A. Szameit, and M. I. Molina, Phys. Rev. Lett. 114, 245503 (2015).

[7] S. Mukherjee, A. Spracklen, D. Choudhury, N. Goldman, P. Öhberg, E. Andersson, and R. R. Thomson, Phys. Rev. Lett. 114, 245504 (2015).

[8] Y. Zong, S. Xia, L. Tang, D. Song, Y. Hu, Y. Pei, J. Su, Y. Li, and Z. Chen, Opt. Express 24, 8877 (2016).

[9] S. Xia, Y. Hu, D. Song, Y. Zong, L. Tang, and Z. Chen, Opt. Lett. 41, 1435 (2016).

[10] S. Weimann, L. Morales-Inostroza, B. Real, C. Cantillano, A. Szameit, and R. A. Vicencio, Opt. Lett. 41, 2414 (2016).

[11] S. Mukherjee and R. R. Thomson, Opt. Lett. 40, 5443 (2015).

[12] B. Real, C. Cantillano, D. López-González, A. Szameit, M. Aono, M. Naruse, S. Kim, K. Wang, and R. A. Vicencio, Sci. Rep. 7, 15085 (2017).

[13] C. Cantillano, S. Mukherjee, L. Morales-Inostroza, B. Real, G. Cáceres-Aravena, C. Hermann-Avigliano, R. R. Thomson, and R. A. Vicencio, New J. Phys. 20, 033028 (2018).
[14] Z. Lin, J.-H. Choi, Q. Zhang, W. Qin, S. Yi, P. Wang, L. Li, Y. Wang, H. Zhang, Z. Sun, L. Wei, S. Zhang, T. Guo, Q. Lu, J.-H. Cho, C. Zeng, and Z. Zhang, Phys. Rev. Lett. 121, 096401 (2018).

[15] G.-B. Jo, J. Guzman, C. K. Thomas, P. Hosur, A. Vishwanath, and D. M. Stamper-Kurn, Phys. Rev. Lett. 108, 045305 (2012).

[16] F. Baboux, L. Ge, T. Jacqmin, M. Biondi, E. Galopin, A. Lemaître, L. Le Gratiet, I. Sagnes, S. Schmidt, H. E. Türeci, A. Amo, and J. Bloch, Phys. Rev. Lett. 116, 066402 (2016).

[17] Y.-X. Xiao, G. Ma, Z.-Q. Zhang, and C. T. Chan, Phys. Rev. Lett. 118, 166803 (2017).

[18] T. Bilitewski and R. Moessner, Phys. Rev. B 98, 235109 (2018).

[19] P. Shukla, Phys. Rev. B 98, 054206 (2018).

[20] V. Goblot, B. Rauer, F. Vicentini, A. Le Boité, E. Galopin, A. Lemaître, L. Le Gratiet, A. Harouri, I. Sagnes, S. Ravets, C. Ciuti, A. Amo, and J. Bloch, Phys. Rev. Lett. 123, 113901 (2019).

[21] T. Biesenthal, M. Kremer, M. Heinrich, and A. Szameit, Phys. Rev. Lett. 123, 183601 (2019).

[22] D. Guzmán-Silva, C. Mejía-Cortés, M. A. Bandres, M. C. Rechtsman, S. Weimann, S. Nolte, M. Segev, A. Szameit, and R. A. Vicencio, New J. Phys. 16, 063061 (2014).

[23] R. A. Vicencio, M. I. Molina, and Y. S. Kivshar, Opt. Lett. 28, 1942 (2003).

[24] D. K. Cambpell, S. Flach, and Y. S. Kivshar, Phys. Today 57(1), 43 (2004).

[25] F. Lederer, G. I. Stegeman, D. N. Christodoulides, G. Assanto, M. Segev, and Y. Silberberg, Phys. Rep. 463, 1 (2008). 
[26] S. Flach and A. Gorbach, Phys. Rep. 467, 1 (2008).

[27] R. A. Vicencio and M. Johansson, Phys. Rev. A 87, 061803(R) (2013).

[28] C. Danieli, A. Maluckov, and S. Flach, Low Temp. Phys. 44, 865 (2018).

[29] M. Johansson, U. Naether, and R. A. Vicencio, Phys. Rev. E 92, 032912 (2015).

[30] P. P. Beličev, G. Gligorić, A. Radosavljević, A. Maluckov, M. Stepić, R. A. Vicencio, and M. Johansson, Phys. Rev. E 92, 052916 (2015).

[31] P. P. Beličev, G. Gligorić, A. Maluckov, M. Stepić, and M. Johansson, Phys. Rev. A 96, 063838 (2017).

[32] B. Real and R. A. Vicencio, Phys. Rev. A 98, 053845 (2018).

[33] B. Pal, Phys. Rev. B 98, 245116 (2018).

[34] A. Szameit, D. Blömer, J. Burghoff, T. Pertsch, S. Nolte, and A. Tünnermann, Appl. Phys. B 82, 507 (2006).

[35] Y. Yamashita, M. Tomura, Y. Yanagi, and K. Ueda, Phys. Rev. B 88, 195104 (2013).

[36] A. Bao, H.-S. Tao, H.-D. Liu, X. Z. Zhang, and W.-M. Liu, Sci. Rep. 4, 6918 (2014).

[37] M. Kargarian and G. A. Fiete, Phys. Rev. B 82, 085106 (2010).

[38] A. Khare, K. Ø. Rasmussen, M. R. Samuelsen, and A. Saxena, J. Phys. A 38, 807 (2005).

[39] J. L. Marin and S. Aubry, Physica D 119, 163 (1998).
[40] M. Johansson and Yu. S. Kivshar, Phys. Rev. Lett. 82, 85 (1999).

[41] A. M. Morgante, M. Johansson, G. Kopidakis, and S. Aubry, Phys. Rev. Lett. 85, 550 (2000).

[42] A. M. Morgante, M. Johansson, G. Kopidakis, and S. Aubry, Physica D 162, 53 (2002).

[43] J. C. Eilbeck, P. S. Lomdahl, and A. C. Scott, Physica D 16, 318 (1985).

[44] L. Casetti and V. Penna, J. Low Temp. Phys. 126, 455 (2002).

[45] M. Machholm, A. Nicolin, C. J. Pethick, and H. Smith, Phys. Rev. A 69, 043604 (2004).

[46] U. Naether, A. J. Martínez, D. Guzmán-Silva, M. I. Molina, and R. A. Vicencio, Phys. Rev. E 87, 062914 (2013).

[47] Lj. Hadžievski, A. Maluckov, M. Stepić, and D. Kip, Phys. Rev. Lett. 93, 033901 (2004).

[48] Yu. S. Kivshar and D. K. Campbell, Phys. Rev. E 48, 3077 (1993).

[49] U. Naether, R. A. Vicencio, and M. Johansson, Phys. Rev. E 83, 036601 (2011).

[50] R. A. Vicencio, B. Real, C. Cantillano, L. Morales-Inostroza, and C. Mejía-Cortés, U.S. Patent No. US2018/0210150A1 (2018).

Correction: Minor changes were made to the captions of Figs. 2, 7, and 9. 